\title{
Design and Research on Amphibious Robot
}

\author{
R.Raffik, S.Karthikeyan, P. Abishekraj, K. Manoj Guha
}

\begin{abstract}
Robots plays a major role in industrial automation and in wide range of applications such as manufacturing, surgery, or handling of hazardous materials. The aim of this research project is to design two wheeled amphibious robot which can travel on both land and water. In this project, a bot is designed with proper balancing with good structured buoyancy effects to make the bot to float without problem. Due to the mobility in water, the robot has been designed with insulation and water proof materials.
\end{abstract} Locomotion, Mobility, Wheels with blades

\section{INTRODUCTION}

Amphibots are the robots which have the mobility in both land and water. These kind of bots will be useful for the surveillance and transportation purposes. In autonomous defense and surveillance applications, the robots has to move in various platforms and surfaces. In ocean sea-shores, the robots has to navigate in rock solid terrains and in sandy beaches. The robot has to move in all directions. The main advantages of the amphibot design are

- More powerful,

- Easy Handling and

- Easy fabrication.

\section{LITERATURE REVIEW}

An amphibious snake robot capable of crawling and swimming is discussed and the experiments were carried out to analyze the mobility and its velocity on various surfaces both in water and on ground. Using these experimental conclusions, we can identify the optimized and fastest locomotion possibilities. Depends on the frequencies, amplitudes, and phase lags of undulatory gaits with larger optimal regions the results are taken in parameter space for the crawling gaits. Mobility in water is much faster than crawling for the same frequencies. With both medium, the fastest locomotion is observed and these results are compared with data from fishes and amphibian snakes [1, 2].

A detailed study about quadruped walking machine with moving configuration was carried out. Normally, the combination of lead screw and linkage mechanism is designed for foot moving configuration. These mechanisms

Revised Manuscript Received on August 14, 2019.

R.Raffik, Department of Mechatronics Engineering, Kumaraguru College of Technology, Coimbatore, Tamil Nadu, India. (E-mail: raffikmts@gmail.com, raffik.r.mce@kct.ac.in)

S.Karthikeyan, Department of Mechanical Engineering, Christian College of Engineering and Technology, Oddanchatram, Tamilnadu, India. (E-mail: mekarthikeyan1@gmail.com)

P. Abishekraj, Department of Mechatronics Engineering, Kumaraguru abishekraj1309@gmail.com)

K. Manoj Guha, Department of Mechatronics Engineering, Kumaraguru College of Technology, Coimbatore, Tamil Nadu, India. (E-mail: 4manojguha97@gmail.com)
Keywords - Biomimetic, Amphibot, Self-balancing, Buoyancy, College of Technology, Coimbatore, Tamil Nadu, India. (E-mail:

will produce left and right deviation or up and down vibration during motion. It's self-balancing status while taking stride is very poor. But the quadruped walking machine design tremendously decreases up and down vibration and left and right deflection of the body. Additionally the machine's capability to visualize the work envelope has been increased. In future, the rotation axis design can be implemented for the design of amphibious robot due to better dust-proof and water proof enhanced designs [3].

The challenges in the designing process of an amphibious robot for various modes of motion was discussed. This robot is inspired by the biomimetics aspects from nature. The fishes and cetaceans uses fins as an efficient propulsive mode in under-water applications. A combination of carangiform or dolphin-like swimming and wheel-like motions is designed for the multi-purpose amphibious propulsive mechanism. Based on the bio inspired design, the robot is designed with a head, multiple identical propellingunits, and a flapping foil, which allows the robot in a flexible manner. It also enables the system to fabricate with reliable maintenance. An embedded control system is incorporated with the robot and corresponding motion control algorithms are addressed. Experiments are carried out to optimize the mobility effectiveness of the mechanism [4].

An amphibious robot is designed to enable the robot to move in shallow-water and difficult terrains. An amphibious probing and surveillance system with camera is structured in such a way that it has to perform many demanding tasks in underwater, various terrains and weather conditions. Experimental are conducted and the results shows that the system is more reliable and efficient [5].

A bio-inspired amphibious robot well-suited for the locomotion on both ground and under water with multimode motion is designed. It concerns about the integration of mechatronics aspects in the design and development of amphibious robot. An ARM based embedded control system with well-established software and hardware designs are formed. The environmental information has been collected and the same is used for the adaptive control in dynamically changing environments.

An amphibious robot with integrated embedded control system with propeller is designed to swim and climb. The control system optimizes mass and thrust of propeller to attain the locomotion in underwater. The design and analysis has been done to calculate the propeller thrust with the help a CAD software and a CFD software. The design optimization was also carried out for the material selection

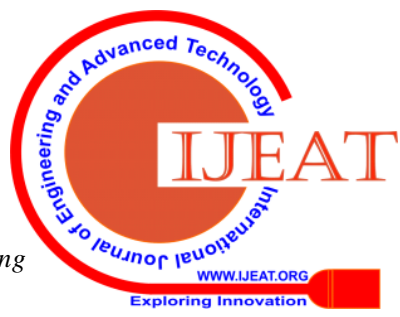


in order to reduce the mass and thereby increases the thrust force. Design of experiments (DOE) has been done and the various parameter affects the efficiency and of the robot has been analyzed [6].

The design and optimization of a bio-inspired amphibious robot has been done specially for the sea beach zone. The autonomous robots has to navigate various terrain surfaces and it has to stabilize itself in dynamic underwater currents. After reaching the beach zone, the loose dry sand requires more energy to move on it. The combination of these locomotion make the system to deploy and operate in defense and civilian missions. [7].

\section{DESIGN METHODOLOGY}

\section{A. Process Flow \& Control of Amphibot}

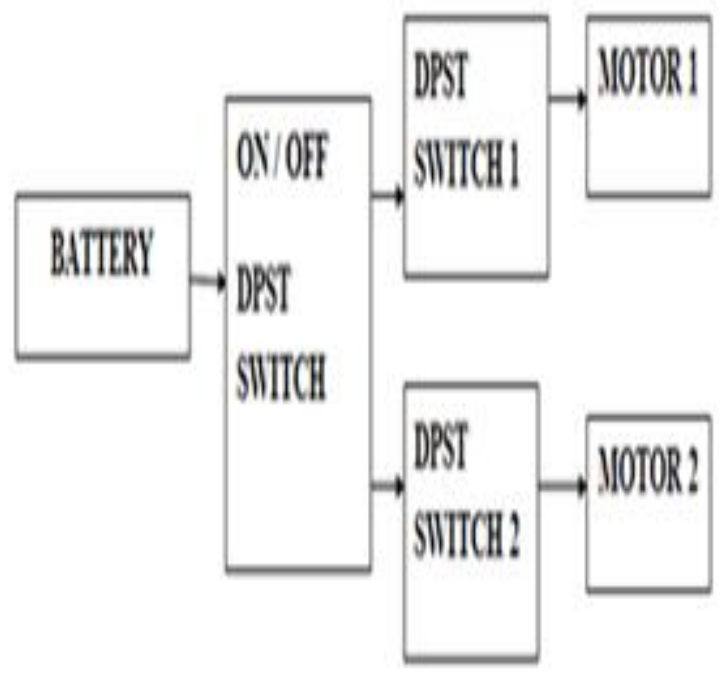

Fig.1. Process Flow and Control of Amphibot

\section{B. Procedure}

This project is designed in such a way that it runs with two wheels on earth. This project contains the following parts control unit, motor, blades and robot model. The robot model has two wheels. Two motors are fixed to run the vehicle. Each wheel is connected to a DC motor. The robot has blade attached to the wheels.

When the motor starts to rotate the wheel will rotate. Multiple blades are attached to both the wheels. Power is supplied to the motor with the help of a $12 \mathrm{v}$ battery. Thus the motion of the robot is achieved on the land. So the amphibot moves from one place to another with help of the motor. The same operation also takes place when the robot is in the water. Blade for swimming is attached to the wheels at an inclined position. This helps the robot to move in water by pushing the water. Thermocol is fixed over the motor assembly which prevents the robot to sink in the water.

The wheels are attached with blades which are used to make the bot to move in water. Bearing is used to mount the motor. Ball bearing is used because when the bot comes from dynamic to static state the body has to be made stable and it should not be in improper position and there comes the play of bearing. Bearing is the key for the bot to make the body to be stable by making the body to come back to normal position.
Wheels are attached to the motor shaft, so as the motor rotate the wheel rotate thus motion is achieved. Wheels are going to be fabricated and it is going to be stick with standard Boe-Bot wheels. Boe-bot wheel is used for coupling and wheels easily. Blades are attached to the wheels which helps the bot to swim on the water. Pedals were attached to the wheels which helps the bot to travel on the water. As the wheels rotate the pedals also rotate so swimming is achieved. The blades are attached in such a way the bot can easily swim in all direction. It is placed perpendicular to the sides of the wheel to push the water thus motion is attained on water. 8 blades are attached so that the effort of peddling on the water is made easy.

A battery and 3 DPST switch are going to be used. Electrical connection was given as shown in the layout diagram. A separate board was made to attach the battery and DPST switch. Thermocol is used due to its very light and good floating characteristics. This material is chosen since it is using buoyancy principle. It has a high insulating efficiency, resistance to moisture, adequate structural strength and dimensional stability makes it easy to use. Improper weight distribution may lead the bot to become unstable. A weight is placed in the middle of the body in order to balance the bot and make it stable when bot is in stationary.

\section{DESIGN AND CALCULATIONS \& RESULTS}

\section{A. Front view without polystyrene sheet}

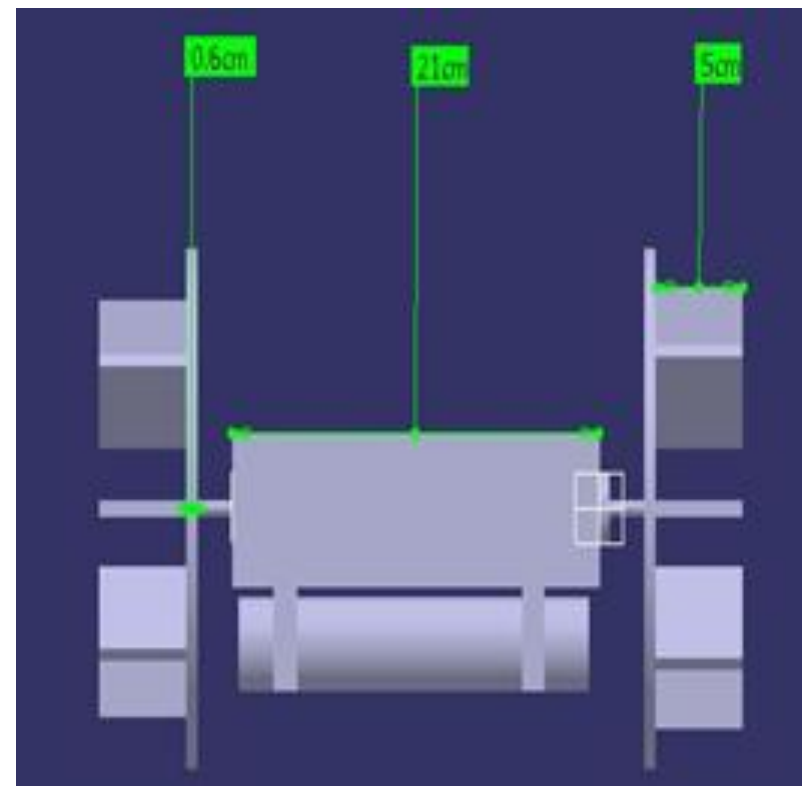

Fig. 2. Front view without polystyrene sheets

Model was designed in CATIA V5. This view shows the 3D image of the amphibot, model was designed taking into consideration of all component dimensions.

\section{B. Wheel design}

This is the front view of the model with dimensions. The blade dimensions, whole body dimensions, wheel thickness are indicated. 


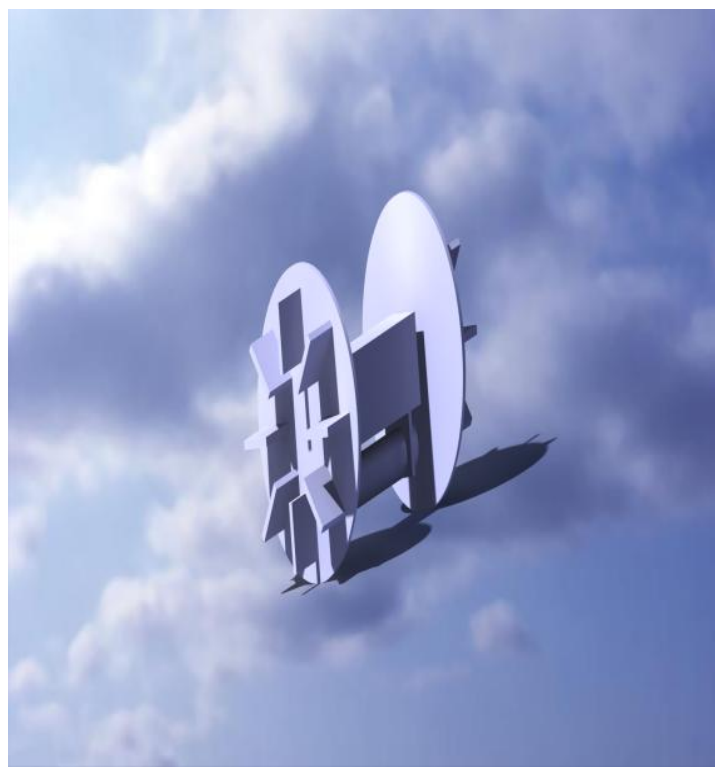

Fig. 3. Isometric view of wheel design

C. Isometric view with polystyrene sheet

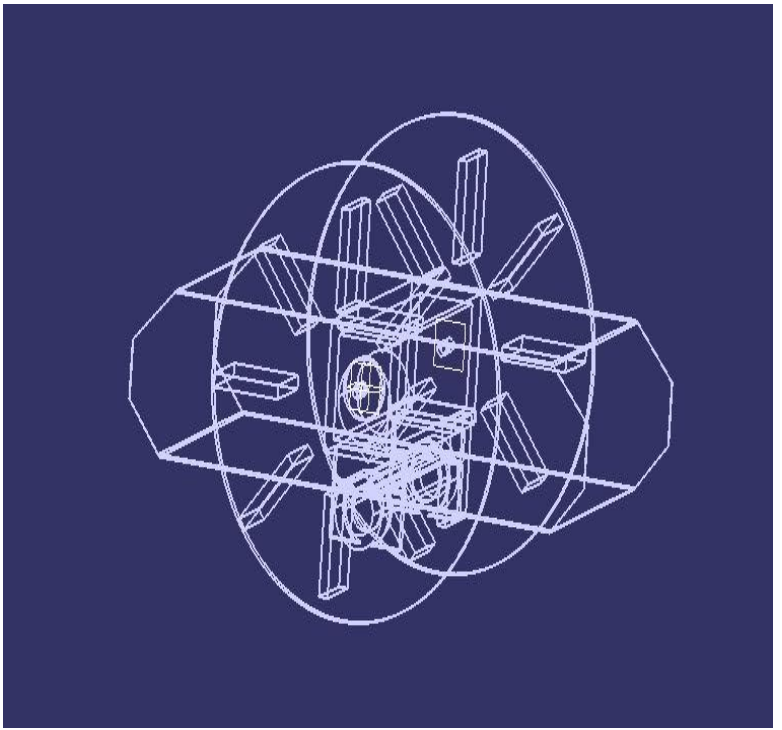

Fig. 4. Isometric view with polystyrene sheets

Total design of the model is represented above, position of the thermocol is shown above.

\section{Design calculations}

\section{1) Motor Rpm:}

Taking into consideration that our bot should travel at a speed (v) of $4 \mathrm{kmph}$,

Velocity $=(4 * 5) / 18$

$\mathrm{V}=1.11 \mathrm{~m} / \mathrm{s}$

Since our bot is an All-Terrain Vehicle, it should have more ground clearance, so larger diameter wheel was need. The wheel with radius(r) $10 \mathrm{~cm}$ was fabricated using plastic.

Radius of the wheel $(\mathrm{r})=10 \mathrm{~cm}=0.1 \mathrm{~m}$

To find the angular velocity

$\mathrm{V}=\mathrm{r} * \omega$

we know $\mathrm{v}$ and $\mathrm{r}$,

$1.11=0.1 * \omega$ (Angular Velocity)

$\omega=11.1 \mathrm{rad} / \mathrm{sec}$

where $\omega=2 \pi \mathrm{N} / 60$

$\mathrm{N}=(\omega * 60) / 2 \pi$
$=(11.1 * 60) /(2 * 3.14)$

$\mathrm{N}=106 \mathrm{RPM} \sim 100 \mathrm{RPM}$

So, from (1) the speed (RPM) of the motor was found

2) Torque calculations:

Over all mass of the bot is $2 \mathrm{~kg} \sim 20 \mathrm{~N}$. RnL, RnR are the two reaction forces acting on left and right wheels as shown in Fig. 5. Length of bot is $21 \mathrm{~cm}$. Center of gravity is acting at the middle $(10.5 \mathrm{~cm})$ since loads are equal on both sides.

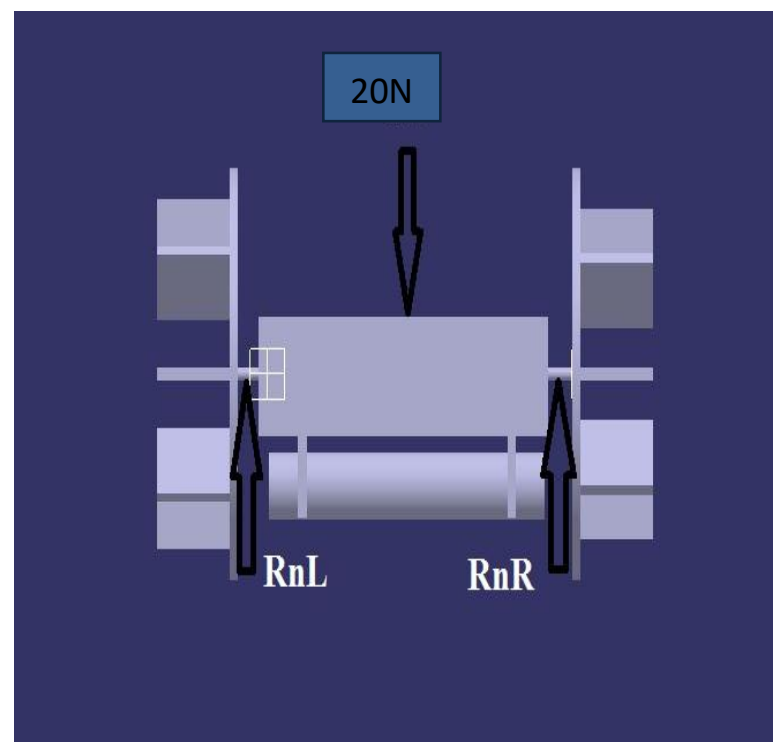

Fig. 5. Front view with load conditions

Taking moment at right wheel (RnR),

$\mathrm{RnL} * 21=20 * 10.5$

$\mathrm{RnL}=210 / 21=10 \mathrm{~N}$

To find frictional force $(\mathrm{F})$,

$\mathrm{F}=\mathrm{Rn} * \mu$

where, $\mu=0.5$

$\mathrm{F}=10 * 0.5=5 \mathrm{~N}$

To find starting Torque

$(\mathrm{T})=\mathrm{F} * \mathrm{r}$

where, $\mathrm{r}=0.1 \mathrm{~m}$

$=5 * 0.1$

Starting Torque $(\mathrm{T})=0.5 \mathrm{Nm}$

From (2) and (3), the required torque of the motor was calculated and motor was selected.

3) Power $(P)$ required

$\mathrm{P}=2 \pi \mathrm{NT} / 60$

$=(2 * 3.14 * 100 * 0.5) / 60$

where, $\mathrm{N}=100 \mathrm{RPM}$ and $\mathrm{T}=0.5 \mathrm{Nm}$

Power $=5 \mathrm{~W}$

E. Components specification

1) DC Motor:

Voltage $=12 \mathrm{~V}$

Speed $=100 \mathrm{RPM}$

Torque $=0.5 \mathrm{Nm}$

Max load current $=800 \mathrm{~mA}$

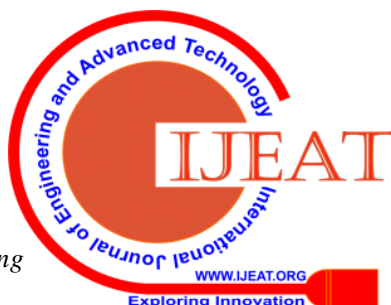




\section{2) Battery}

Voltage $=12 \mathrm{~V}$

Current $=1.3 \mathrm{~A}$

\section{F. Fabrication of Amphibot}

The step by step fabrication process and the completed design model with the necessary attachments and mountings. As per the CAD model shown in Fig. 2 and Fig.4, all the dimensions were marked in the plastic body and all pieces were cut using hacksaw blade. The body panel and wheel with blade like structures was made to stick with each other using araldite, in some places plastic was melted and made to stick with each other as shown in Fig. 3. Bearing was mounted in the plastic body by melting it and made to fit firmly as shown in Fig. 6 and Fig. 7. Bearing are the supporting members to support the motor firmly.

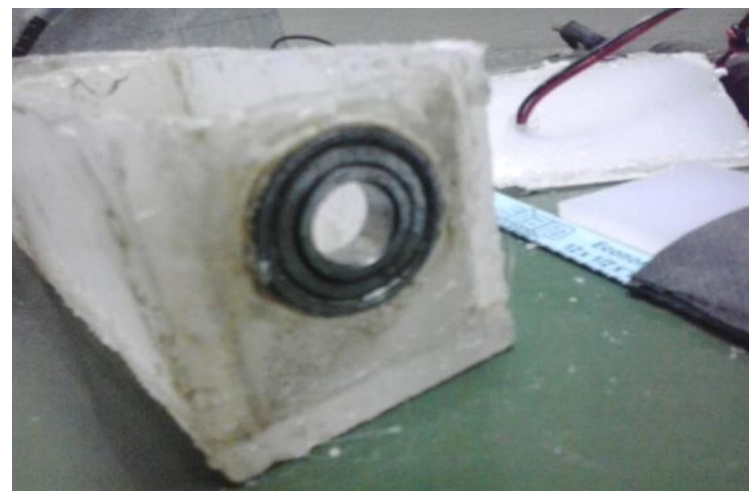

Fig. 6. Bearings attachments

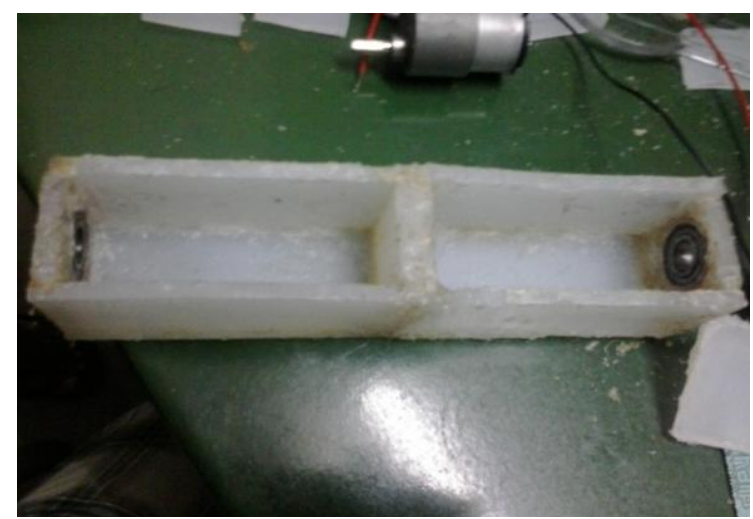

Fig. 7. Two sections of bot

Rubber was used in order to make the bot as leak proof. Rubbers were attached to the circumference of the tire so that there is good friction between the surface and the tire. These rubbers were cut from old vehicle tube. Polystyrene is very light weight and also very good floating material. Polystyrene pads was cut in a shape as shown in Fig. 8, that can be attached with the bot to improve the buoyancy effect and floating capacity of the bot, i.e. polystyrene pads is cut in a shape to improve the hydro dynamics of the bot.

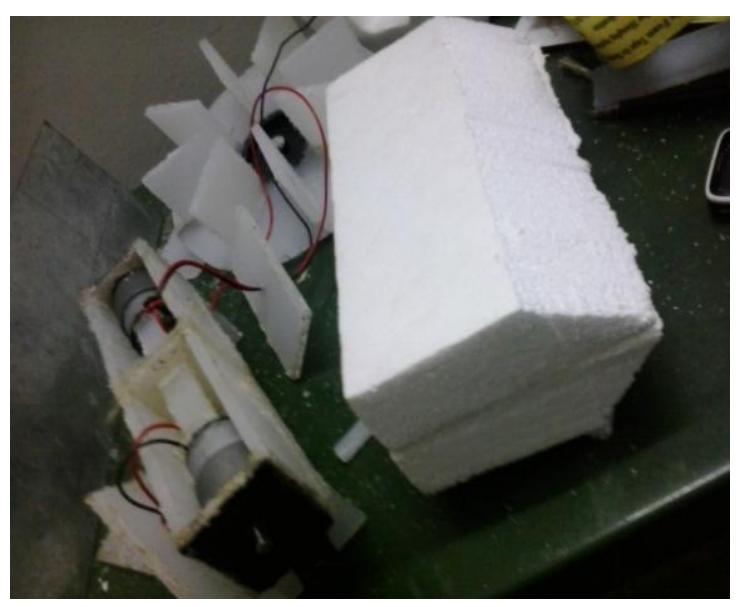

Fig. 8. Motor attachments with hydro-dynamically cut polystyrene sheets

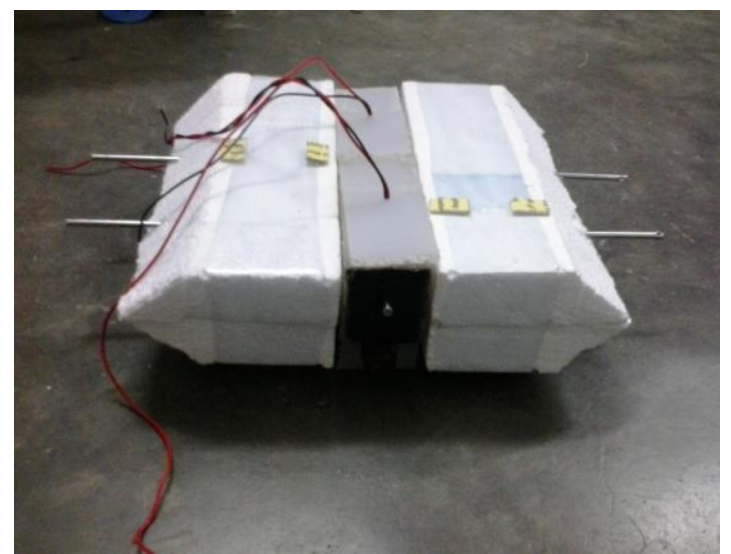

Fig. 9. Water resistive material and polystyrene sheets fixed to bot

Polystyrene (Thermocol) pads was attached to the bot using a rod, the rod makes the polystyrene pads to rigidly attach with the bot as shown in Fig. 9. It acts as the supporting member for the thermocol. Thus the fabrication of the bot was successfully done and the same is shown in Fig. 10. It is made to travel on both land and water. The water resistant materials and glues are used to protect the motors from the water and thereby avoids the shortcircuiting of electrical connections.

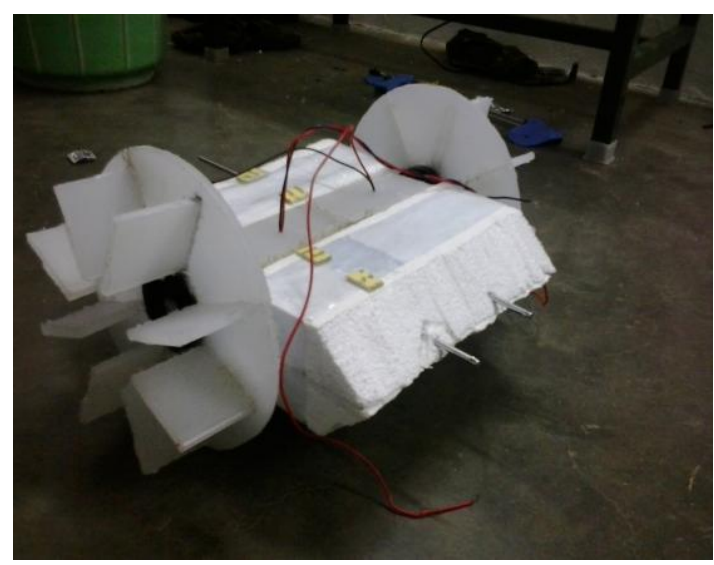

Fig. 10. PAL - The Amphibot

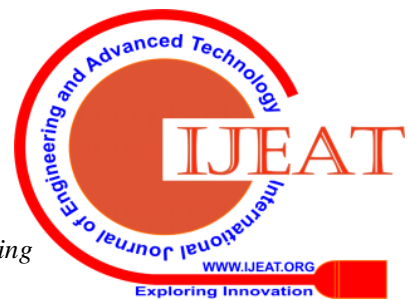




\section{CONCLUSION}

A new design for amphibious robot has been discussed in this paper. Depends on the load conditions and requirements, the motors and structures are selected. In order to increase the buoyancy of the bot, the supporting rods and polystyrene sheets are designed and attached to the bot to improve the hydro-dynamic motion control. Proper sealings are done to resist the water to enter inside the drive control part. The bot is made to move in water and land and the velocity of bot are measured on both the mediums. In land without any obstacles, the bot moves about with a velocity of $1 \mathrm{~m} / \mathrm{sec}$. But in the water, it moves with a velocity around $0.4 \mathrm{~m} / \mathrm{sec}$.

\section{REFERENCES}

1. A. Crespi, A. Badertscher, A. Guignard, A. Ijspeert, "AmphiBot I: an amphibious snake-like robot", Robotics and Autonomous Systems, vol. 50, 2005, pp. 163-175.

2. A. Crespi, A. Ijspeert, "AmphiBot II: an amphibious snake robot that crawls and swims using a central pattern generator", Proceedings of the 9th Fig. 9. International Conference on Climbing and Walking Robots, 2006, pp. 19. 27.

3. Y. Lin, Y. Hsieh, C. Yang, K. Hang and K. Lee, "The Level Moving Configuration of A Quadruped Walking Machine", IECON 2007 - 33rd Annual Conference of the IEEE Industrial Electronics Society, Taipei, 2007, pp. 471-476.

4. Q. Yang, J. Yu, M. Tan and W. Wang, "Preliminary development of a biomimetic amphibious robot capable of multi-mode motion," 2007 IEEE International Conference on Robotics and Biomimetics (ROBIO), Sanya, 2007, pp. 769774.

5. Ying Tang and Hong Zhang, "An Interactive Mobile Amphibious Probing and Surveillance System," 2008 International Conference on Information and Automation, Changsha, 2008, pp. 441-445.

6. Rui Ding, J. Yu, Q. Yang, Xiaolei Hu and M. Tan, "Platformlevel design for a biomimetic amphibious robot," 2008 IEEE International Conference on Robotics and Biomimetics, Bangkok, 2009, pp. 977-982.

7. A. S. Boxerbaum, M. A. Klein, R. Bachmann, R. D. Quinn, R. Harkins and R. Vaidyanathan, "Design of a semi-autonomous hybrid mobility surf-zone robot," 2009 IEEE/ASME International Conference on Advanced Intelligent Mechatronics, Singapore, 2009, pp. 974-979. 